\title{
Perfect Lattice Actions for the Gross-Neveu Model
}

\author{
Wolfgang Bietenholz ${ }^{\mathrm{a}}$, Erich Focht ${ }^{\mathrm{b}}$ and Uwe-Jens Wiese ${ }^{\mathrm{a} *}$ \\ ${ }^{a}$ Center for Theoretical Physics, Massachusetts Institute of Technology(MIT) \\ Cambridge, Massachusetts 02139, USA \\ ${ }^{\mathrm{b}}$ Institute for Theoretical Physics E, RWTH-Aachen, 52074 Aachen, Germany \\ HLRZ c/o KFA Jülich, 52425 Jülich, Germany
}

\begin{abstract}
We apply the method of Hasenfratz and Niedermayer to analytically construct perfect lattice actions for the Gross-Neveu model. In the large $N$ limit these actions display an exactly perfect scaling, i.e. cut-off artifacts are completely eliminated even at arbitrarily short correlation length. Also the energy spectrum coincides with the spectrum in the continuum and continuous translation and rotation symmetries are restored in physical observables. This is the first (analytic) construction of an exactly perfect lattice action at finite correlation length.
\end{abstract}

\section{Introduction}

The worst systematic errors in lattice simulations are due to cut-off artifacts. For fermionic systems they are typically of $O(a)$, where $a$ is the lattice spacing. For a long time, the attempts to improve the lattice action such that the artifacts are dampened, yielded only a limited success. Last year, P. Hasenfratz and F. Niedermayer proposed a new method for approximating perfect actions (actions free of cut-off effects) for asymptotically free theories [1]. It might have the potential for a break-through which enables us to solve lattice QCD.

First we note that the fixed point actions (FPAs) - obtained from iterated block spin renormalization group transformations (RGTs) on a critical surface (see e.g. [2]) - are perfect actions. However, since the correlation length $\xi$ diverges there, they cannot be used in simulations. Kogut and Wilson [3] noticed that perfect actions exist also off the critical surface. They lie on 'renormalized trajectories' (RTs) in parameter space, which cross the critical surface in a fixed point. The direction of the tangent there corresponds to a relevant (or marginal) direction. If we move slightly

\footnotetext{
*Based on two talks presented by E.F. and W.B. Work supported by Deutsche Forschungsgemeinschaft (D.F.G.) and U.S. Department of Energy (D.O.E.) under cooperative research agreement DE-FC02-94ER40818.
}

away from a fixed point in a relevant direction and perform RGTs, then we follow the RT. Thus all points on it are related to the vicinity of the fixed point without occurrence of irrelevant terms.

For asymptotically free theories, there is only one (weakly) relevant (to leading order marginal) direction. Hasenfratz and Niedermayer propose to follow this direction down to a $\xi$ short enough for simulation, i.e. to approximate the RT linearly. The points on this tangent are referred to as 'perfect classical actions' and there is hope that they still have very small cut-off artifacts. The virtue of asymptotically free theories (in this context) is that their FPAs can be determined classically.

The pilot project of Hasenfratz and Niedermayer dealt with the $O(3)$ nonlinear $\sigma$ model in $d=2$ and was a full success. Even on a lattice as small as $5 \times 5$ cut-off effects were numerically not visible any more.

The final goal is of course QCD. A collaboration is presently studying the pure $S U(3)$ gauge theory in this respect [⿶] .

Here we present results for the 2d Gross-Neveu model [5], which turned out to be a good testing ground for the method when applied to fermionic systems. 


\section{Fixed point actions for free lattice fermions}

First, one of us found a line of FPAs for free Wilson fermions [6] which also applies to a number of other lattice fermions (SLAC, Rebbi, etc., see [7]). However, this set does not include the staggered fermions which we are going to use here. Their main advantage is the remnant $U(1) \otimes U(1)$ symmetry which persists at free, local fixed points [8] We perform RGTs according to the prescription in [9] i.e. we attach pseudo flavors to the corners of (disjoint) $2 \times 2$ blocks and build block spins such that the pseudo flavors don't mix. Therefore an odd blocking factor is needed; we chose it to be 3 . Then we have intersecting $9 \times 9$ blocks, whose centers form a lattice of spacing 3 and which are occupied by alternating pseudo flavors. Every point in a block with the same pseudo flavor as the center contributes to its block spin which lives again on the block center. Then we rescale coordinates and fields.

We take a lattice of spacing $1 / 2$. For technical reasons we initially put the fermionic pseudo flavors $\bar{\chi}^{1}, \ldots \bar{\chi}^{4}, \chi^{1} \ldots \chi^{4}$ (one-component Grassmann variables) on the centers $x$ of disjoint $2 \times 2$ blocks (spacing 1). Later the fermionic variables are moved to the corners (in [8] we proceeded differently). For the action after a number of RGTs we make the general ansatz

$S[\bar{\chi}, \chi]=\sum_{x, y} \sum_{i, j=1}^{4} \bar{\chi}_{x}^{i} \rho^{i j}(x-y) \chi_{y}^{j}$.

$S$ is invariant against translations by integers (but not half integers) along the coordinate axes. The matrix for the fermions shifted to the corners is (in momentum space) denoted by $\tilde{\rho}(k)$. Its form is restricted by charge conjugation, $U(1) \otimes U(1)$ symmetry and half integer translations. Its structure, resp. the one of its inverse $\tilde{\alpha}(k)=\tilde{\rho}^{-1}(k)$ is

$$
\tilde{\alpha}(k)=\left(\begin{array}{cccc}
0 & \tilde{\alpha}_{1}(k) & \tilde{\alpha}_{2}(k) & 0 \\
\tilde{\alpha}_{1}(k) & 0 & 0 & -\tilde{\alpha}_{2}(k) \\
\tilde{\alpha}_{2}(k) & 0 & 0 & \tilde{\alpha}_{1}(k) \\
0 & -\tilde{\alpha}_{2}(k) & \tilde{\alpha}_{1}(k) & 0
\end{array}\right) .
$$

${ }^{2}$ For Wilson fermions the full chiral symmetry may be preserved, but then the FPA becomes non local, in agreement with the Nielsen-Ninomiya theorem.
Starting from the standard action with $\tilde{\alpha}_{\mu}(k)=$ $i \hat{k}_{\mu} / \hat{k}^{2}$ (in lattice notation: $\hat{k}_{\mu} \doteq 2 \sin \left(k_{\mu} / 2\right)$ ), we arrive at the fixed point given by

$$
\begin{aligned}
\tilde{\alpha}_{\mu}^{*}(k) & =i \sum_{l \in \mathbb{Z}^{2}} \frac{k_{\mu}+2 \pi l_{\mu}}{(k+2 \pi l)^{2}}(-)^{l_{\mu}} \prod_{\nu}\left(\frac{\hat{k}_{\nu}}{k_{\nu}+2 \pi l_{\nu}}\right)^{2} \\
& +\frac{9}{8 c} i \hat{k}_{\mu} .
\end{aligned}
$$

Here a renormalization parameter in the blocking step had to be chosen in agreement with the dimension of $\chi$. The case $c \rightarrow \infty$ corresponds to a $\delta$-function blocking RGT, but we may add a 'smearing term' suppressed by $1 / c$, which does not break any symmetry and which helps to optimize the locality, see [8].

\section{The Gross-Neveu model}

Our main interest was to gain experience with interacting fermionic models, the simplest asymptotically free one being the Gross-Neveu model in two dimensions (GN). It is a model with 4-Fermi interaction which can be linearized by introducing a real valued auxiliary field $\Phi$. With $G$ being the usual 4-Fermi coupling the continuum Euclidean action for $N$ flavors is

$\frac{1}{G} S[\bar{\chi}, \chi, \Phi]=\frac{1}{G} \int d^{2} x \sum_{i=1}^{N}\left(\bar{\chi}^{i} \not \partial \chi^{i}+\bar{\chi}^{i} \chi^{i} \Phi\right)+\frac{1}{2} \Phi^{2}$.

The $O(2 N) \times Z(2)$ symmetry of the model is broken spontaneously to $O(2 N)$ and a fermion mass $m_{f}$ is generated dynamically.

In a sensible lattice formulation of the GN model with staggered fermions and Yukawa coupling, the scalars live on the centers of the fermionic lattice, i.e. they couple equally to the 4 pseudo flavors at one plaquette and their spacing is $1 / 2$. To keep a Gaussian form we do not integrate out $\Phi$ but block it, simultaneous to the fermionic blocking. For this we use the same pattern: we block them as if they also had pseudo flavors, which must not be mixed, see fig.1.

\section{Small field approximation}

For the moment we only include the first order in $\Phi$ and let $N=2$. Then we parametrize a 


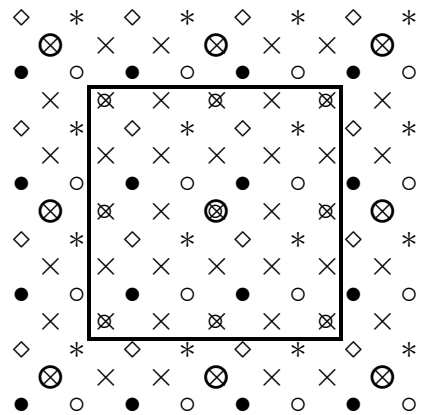

Figure 1. Geometry of the scalar (crosses) and fermionic variables (different symbols for the pseudo flavors). The nine auxiliary field variables marked with a small circle contribute to the block variable in the center of the box.

general ansatz for the action as:

$$
\begin{aligned}
& \frac{1}{G} S[\bar{\chi}, \chi, \Phi]=\frac{1}{G}\left\{\sum_{x, y} \sum_{i, j} \bar{\chi}_{x}^{i} \rho^{i j}(x-y) \chi_{y}^{j}\right. \\
& \left.\quad+\frac{1}{2} \sum_{z} \Phi_{z}^{2}+\sum_{x, y, z} \sum_{i, j} \bar{\chi}_{x}^{i} \sigma^{i j}(x-z, y-z) \chi_{y}^{j} \Phi_{z}\right\} .
\end{aligned}
$$

where $z$ runs over the cell centers (spacing $1 / 2$ ). For the standard action in momentum space, shifting the fermions to the block corners takes $\sigma$ to the form

$$
\begin{aligned}
\tilde{\sigma}(p, q) & =\left(\begin{array}{cccc}
\tilde{\sigma}_{0} & 0 & 0 & -\tilde{\sigma}_{3} \\
0 & \tilde{\sigma}_{0} & \tilde{\sigma}_{3} & 0 \\
0 & -\tilde{\sigma}_{3} & \tilde{\sigma}_{0} & 0 \\
\tilde{\sigma}_{3} & 0 & 0 & \tilde{\sigma}_{0}
\end{array}\right), \\
\tilde{\sigma}_{0} & =\frac{\hat{p}_{\mu} \hat{q}_{\mu}}{\hat{p}^{2} \hat{q}^{2}} \prod_{\nu} \cos \left(\left(p_{\nu}+q_{\nu}\right) / 4\right) \\
\tilde{\sigma}_{3} & =\frac{\hat{p}_{1} \hat{q}_{2}-\hat{p}_{2} \hat{q}_{1}}{\hat{p}^{2} \hat{q}^{2}} \prod_{\nu} \cos \left(\left(p_{\nu}+q_{\nu}\right) / 4\right)
\end{aligned}
$$

The fixed point condition forces us to apply a $\delta$ blocking in the scalar sector. Doing so we arrive at a fixed point, where $\sigma$ still has the same structure and its elements become:

$$
\begin{aligned}
\tilde{\sigma}_{0}^{*} & =\sum_{l, m \in \mathbb{Z}^{2}} \sum_{i} \frac{p_{\mu}^{(l, i)} q_{\mu}^{(m, i)}}{\left(p^{(l, i)}\right)^{2}\left(q^{(m, i)}\right)^{2}} \\
& \times \prod_{\nu}(-)^{l_{\nu}+m_{\nu}+i_{\nu}} \frac{\hat{p}_{\nu} \hat{q}_{\nu}(p \widehat{+q})_{\nu}}{p_{\nu}^{(l, i)} q_{\nu}^{(m, i)}\left(p_{\nu}^{(l, i)}+q_{\nu}^{(m, i)}\right)}
\end{aligned}
$$

$$
\begin{aligned}
\tilde{\sigma}_{3}^{*} & =\sum_{l, m \in \mathbb{Z}^{2}} \sum_{i} \frac{\epsilon_{\mu \rho} p_{\mu}^{(l, i)} q_{\rho}^{(m, i)}}{\left(p^{(l, i)}\right)^{2}\left(q^{(m, i)}\right)^{2}} \\
& \times \prod_{\nu}(-)^{l_{\nu}+m_{\nu}} \frac{\hat{p}_{\nu} \hat{q}_{\nu}(p \widehat{+q})_{\nu}}{p_{\nu}^{(l, i)} q_{\nu}^{(m, i)}\left(p_{\nu}^{(l, i)}+q_{\nu}^{(m, i)}\right)}
\end{aligned}
$$

with $p_{\mu}^{(l, i)}=p_{\mu}+4 \pi l_{\mu}+2 \pi i_{\mu}$ and $i_{\mu} \in\{0,1\}$. The $4 \pi$ antiperiodicity of these elements agrees with the central positions of the scalars. Thus we have identified the direction of the RT out of the critical surface, but only in the parameter subspace which is accessible in the framework of the small field approximation.

\section{The large $N$ limit}

In general one can hardly go beyond the small field approximation to determine the functional $S^{*}[\bar{\chi}, \chi, \Phi]$ analytically. An exception is the limit $N \rightarrow \infty$. There the scalar field freezes to a constant field $\Phi_{0}$ and we can calculate $S^{*}\left[\bar{\chi}, \chi, \Phi_{0}\right]$ nonperturbatively. We parametrize a general ansatz as

$$
\begin{aligned}
& S\left[\bar{\chi}, \chi, \Phi_{0}\right]= \\
& \frac{1}{(2 \pi)^{2}} \int_{B} d^{2} k \bar{\chi}(-k)[\rho(k)+\lambda(k) \mathbb{1}] \chi(k)+\frac{1}{2} \Phi_{0}^{2} V
\end{aligned}
$$

where $B$ is the Brillouin zone and $V$ is the spacetime volume factor (actually infinite). For the standard action $\rho$ is the same as for free fermions and $\lambda(k)=\Phi_{0}$. We define $\tilde{\alpha}(k)+\beta(k) \mathbb{1} \doteq[\tilde{\rho}(k)+$ $\lambda(k) \mathbb{1 1}]^{-1}$ (again the tilde corresponds to fermions shifted to the plaquette corners; for $\lambda$ and $\beta$ there is no change). Still $\tilde{\rho}$ and $\tilde{\alpha}$ keep the structure given in (2) and we obtain at the fixed point

$$
\begin{aligned}
\tilde{\alpha}_{\mu}^{*}(k)= & i \sum_{l \in \mathbb{Z}^{2}} \frac{k_{\mu}+2 \pi l_{\mu}}{(k+2 \pi l)^{2}+\Phi_{0}^{2}}(-1)^{l_{\mu}} \\
& \times \prod_{\nu}\left(\frac{\hat{k}_{\nu}}{k_{\nu}+2 \pi l_{\nu}}\right)^{2}+\frac{9}{8 c} i \hat{k}_{\mu}, \\
\beta^{*}(k)= & \sum_{l \in \mathbb{Z}^{2}} \frac{\Phi_{0}}{(k+2 \pi)^{2}+\Phi_{0}^{2}} \prod_{\nu}\left(\frac{\hat{k}_{\nu}}{k_{\nu}+2 \pi l_{\nu}}\right)^{2} .
\end{aligned}
$$

Here the small field approximation allows to include small non-zero modes of $\Phi(k)$ and may be used if one is interested e.g. in $1 / N$ effects. The result to the first order in $\Phi(k \neq 0)$ is given in 
[5]. The occurring matrix $\tilde{\sigma}^{*}(p, q)$ is more general than the one given in (6), because we expand around any $\Phi_{0}$ now, whereas (5) describes a particular expansion around $\Phi_{0}=0$.

\section{The perfect operator for the chiral con- densate}

Let's perturb $S^{*}$ a little with some operator $X$ :

$S_{j}[\bar{\chi}, \chi, \Phi]=S^{*}[\bar{\chi}, \chi, \Phi]+j X[\bar{\chi}, \chi, \Phi]$,

where $j \ll 1$. We call $X$ a perfect operator and denote it by $X^{*}$ if it is an eigenfunctional of the RGT to $O(j)$

$$
\begin{aligned}
S_{j}^{\prime}\left[\bar{\chi}^{\prime}, \chi^{\prime}, \Phi^{\prime}\right]= & S^{*}\left[\bar{\chi}^{\prime}, \chi^{\prime}, \Phi^{\prime}\right] \\
& +j \gamma X^{*}\left[\bar{\chi}^{\prime}, \chi^{\prime}, \Phi^{\prime}\right]+O\left(j^{2}\right) .
\end{aligned}
$$

The operator is relevant if $\gamma>1$. This is the case for the chiral condensate (with standard operator $\left.\sum_{x} \bar{\chi}_{x} \chi_{x}\right)$ : for dimensional reasons $\gamma$ coincides with the block factor.

We specialize again to $N \rightarrow \infty, \Phi \rightarrow \Phi_{0}$ and also parametrize $X$ as:

$X\left[\bar{\chi}, \chi, \Phi_{0}\right]=\frac{1}{(2 \pi)^{2}} \int_{B} d k \bar{\chi}(-k)[\mu(k)+\nu(k) \mathbb{1}] \chi(k)$,

where $\mu$ has the structure of $\rho$ and $\alpha$. From the above condition (10) for $X^{*}$ we obtain (again shifting the fermions to the block corners)

$$
\begin{aligned}
\tilde{\mu}_{\mu}^{*}(k) & =\partial_{\Phi_{0}} \frac{\tilde{\alpha}_{\mu}^{*}(k)}{\tilde{\alpha}_{\nu}^{*}(-k) \tilde{\alpha}_{\nu}^{*}(k)+\beta^{*}(-k) \beta^{*}(k)}, \\
\nu^{*}(k) & =\partial_{\Phi_{0}} \frac{\beta^{*}(k)}{\tilde{\alpha}_{\nu}^{*}(-k) \tilde{\alpha}_{\nu}^{*}(k)+\beta^{*}(-k) \beta^{*}(k)} .
\end{aligned}
$$

(Omitting $O\left(\Phi_{0}\right)$ we observe that $X^{*}$ is very local.)

\section{The gap equation}

Now we forget about all small field approximations, let $N \rightarrow \infty$ keeping $g=G N$ fixed and exploit our exact result (8). We make use of the dimensional transmutation due to the spontaneous symmetry breaking $U(N / 2) \otimes Z(2) \rightarrow U(N / 2)$, which gives rise to a fermion mass $m_{f}$. We introduce an effective potential $V_{\text {eff }}$ by

$e^{-V_{e f f}\left(\Phi_{0}\right) V}=\int D \bar{\chi} D \chi e^{-\frac{1}{G} S\left[\bar{\chi}, \chi, \Phi_{0}\right]}$.
The condition for $V_{\text {eff }}$ to be minimal yields the gap equation

$2 \Phi_{0}=\frac{1}{(2 \pi)^{2}} \int_{B} d^{2} k g \ln \operatorname{det} \mathcal{M}\left(k, \Phi_{0}\right)$,

$\mathcal{M}=-\frac{1}{G}[\tilde{\rho}+\lambda \mathbb{1}]$ being the fermion matrix for one set of staggered fermions. For the standard action the gap equation takes the form

$\frac{1}{g}=\frac{2}{(2 \pi)^{2}} \int_{B} d^{2} k \frac{1}{\hat{k}^{2}+\Phi_{0}^{2}}$.

obtained and discussed before in [10]. For the FPA it reads

$$
\begin{aligned}
\Phi_{0}= & \frac{1}{(2 \pi)^{2}} \int_{B} d^{2} k g \partial_{\Phi_{0}} \ln \left[\tilde{\alpha}_{\mu}^{*}(-k) \tilde{\alpha}_{\mu}^{*}(k)\right. \\
& \left.+\beta^{*}(-k) \beta^{*}(k)\right] .
\end{aligned}
$$

First we investigate asymptotic scaling of $\Phi_{0}$, see fig.2. Asymptotic scaling corresponds to linear curves. We see that asymptotic scaling for the FPA is clearly better, but still not what one would call 'perfect'. As an alternative consideration of asymptotic scaling we compare in fig. 3 the $\beta$ functions referring to the fermion mass, which we will derive below. It shows the same feature. (Asymptotic scaling is given by: $a m_{f} \propto e^{-\pi / g} \Rightarrow \beta=$ $-m_{f} \partial_{m_{f}} g=-g / \pi^{2}$.) At $\xi=1$ the FPA's $\beta$ function already agrees with this up to 5 percent, but its behavior is still not perfect.

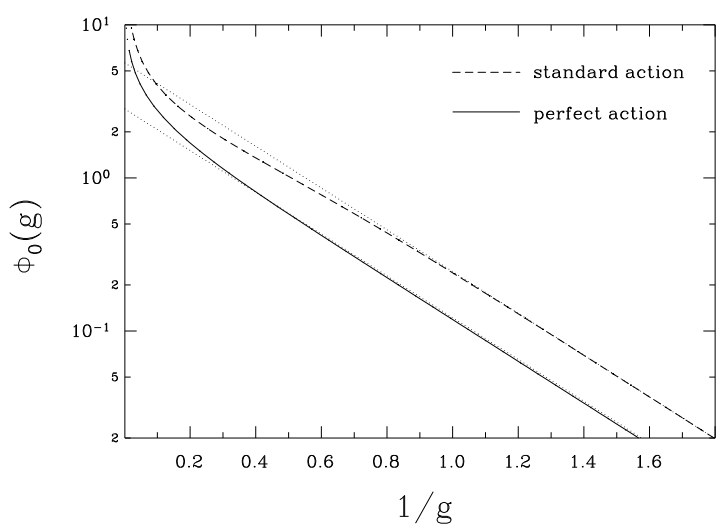

Figure 2. Asymptotic scaling behavior of $\Phi_{0}(g)$. 


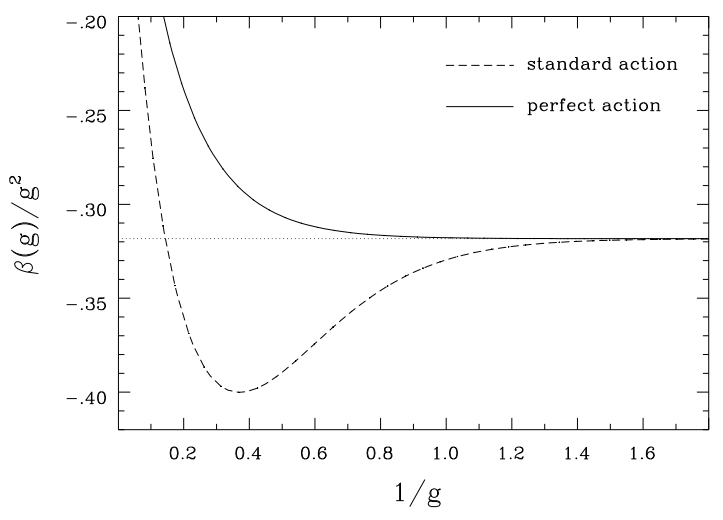

Figure $3 . \beta(g) / g^{2}$ versus $1 / g$.

However, this doesn't mean very much. Asymptotic scaling is not really physical since it involves the bare coupling (at cut-off scale). Actually meaningful are dimensionless ratios.

\section{The scaling behavior}

Such a ratio shall be constructed now to discuss the scaling behavior of the standard action, the small field approximation, and finally of the FPA. First we derive $m_{f}$ from the exponential decay of the correlation function. We put the system in a spatial volume $L$ and consider $x_{2}$ as Euclidean time. The correlation function becomes

$$
\begin{aligned}
& \left\langle\bar{\chi}\left(-k_{1}\right)_{0} \chi\left(k_{1}\right)_{x_{2}}\right\rangle= \\
& \frac{L N}{4 \pi} \int_{-\pi}^{\pi} d k_{2} \operatorname{Tr} \mathcal{M}^{-1}\left(k, \Phi_{0}\right) e^{i k_{2} x_{2}} .
\end{aligned}
$$

For the standard action this turns into

$$
-\frac{L}{\pi} g \Phi_{0} \int_{-\pi}^{\pi} d k_{2} \frac{e^{i k_{2} x_{2}}}{\hat{k}^{2}+\Phi_{0}^{2}} \doteq C\left(k_{1}\right) e^{-E\left(k_{1}\right) x_{2}} .
$$

At the pole of the integrand we find the energy spectrum:

$$
\left[2 \sinh \left(E\left(k_{1}\right) / 2\right)\right]^{2}=\hat{k}_{1}^{2}+\Phi_{0}^{2}
$$

We observe cut-off artifacts of $O\left(a^{2}\right)$. We identify $m_{f}=E(0)$ such that

$2 \sinh \left(m_{f} / 2\right)=\Phi_{0}$.
Now we consider the chiral condensate

$$
\langle\bar{\chi} \chi\rangle=\frac{1}{(2 \pi)^{2}} \frac{N}{2} \int_{B} d^{2} k \operatorname{Tr} \mathcal{M}^{-1}\left(k, \Phi_{0}\right) .
$$

For the standard action the gap equation (14) leads to $\langle\bar{\chi} \chi\rangle=-\Phi_{0}$. Therefore

$$
\left.\frac{\langle\bar{\chi} \chi\rangle}{m_{f}}\right|_{\text {standard }}=-\frac{2 \sinh \left(m_{f} / 2\right)}{m_{f}} .
$$

In the continuum limit, this ratio must become constant, and indeed it does, since: $\xi=m_{f}^{-1} \rightarrow$ $\infty$ (in lattice units). For finite $\xi$, however, we have artifacts of $O\left(a^{2}\right)$. The $Z(2)$ symmetry shields the system from $O(a)$ artifacts.

We don't go through the corresponding formulae for the small field approximation here but just quote the result that the scaling artifacts remain of $O\left(a^{2}\right)$ [5]. There is a slight improvement on the $O\left(a^{2}\right)$ level, but this is not a great progress.

Finally we consider the dispersion relation for the FPA:

$$
\begin{aligned}
& \left\langle\bar{\chi}\left(-k_{1}\right)_{0} \chi\left(k_{1}\right)_{x_{2}}\right\rangle= \\
& -\frac{L}{\pi} g \Phi_{0} \int_{-\pi}^{\pi} d k_{2}\left\{\sum_{l \in \mathbb{Z}^{2}} \frac{e^{i k_{2} x_{2}}}{(k+2 \pi l)^{2}+\Phi_{0}^{2}}\right. \\
& \left.\prod_{\nu}\left(\frac{\hat{k}_{\nu}}{k_{\nu}+2 \pi l_{\nu}}\right)^{2}\right\} \\
= & -\frac{L g \Phi_{0}}{\pi} \int_{-\infty}^{\infty} d k_{2}\left\{\sum_{l_{1} \in \mathbb{Z}} \frac{e^{i k_{2} x_{2}}}{\left(k_{1}+2 \pi l_{1}\right)^{2}+k_{2}^{2}+\Phi_{0}^{2}}\right. \\
& \left.\left(\frac{\hat{k}_{1}}{k_{1}+2 \pi l_{1}} \cdot \frac{\hat{k}_{2}}{k_{2}}\right)^{2}\right\} \\
\doteq & \sum_{l_{1} \in \mathbb{Z}} C\left(k_{1}+2 \pi l_{1}\right) \exp \left(-E\left(k_{1}+2 \pi l_{1}\right) x_{2}\right)
\end{aligned}
$$

For each $l_{1}$ a pole contributes to the correlation function and hence

$$
E^{2}\left(k_{1}+2 \pi l_{1}\right)=-k_{2}^{2}=\left(k_{1}+2 \pi l_{1}\right)^{2}+\Phi_{0}^{2} .
$$

This is the exact continuum energy spectrum. It is remarkable that it is obtained although $k_{1} \in$ ]$-\pi, \pi]$. Here we also observe the restoration of continuous rotational symmetry resp. Lorentz invariance (which can not be seen directly in $\tilde{\alpha}(k)$.) Moreover also the continuous translation invariance is restored (note that $k_{1}+2 \pi l_{1}$ covers the whole real axis). 
Using the perfect operator for the chiral condensate (11) and inserting the FPA's gap eq. (15), we obtain also for the FPA 3

$\langle\bar{\chi} \chi\rangle=-\Phi_{0}$

Now the dimensionless ratio becomes

$\left.\frac{\langle\bar{\chi} \chi\rangle}{m_{f}}\right|_{\mathrm{FPA}}=-1$,

for any $\xi$, i.e. we have perfect scaling.

The result that in the FPA cut-off effects are completely eliminated means that the perfect classical actions are situated on the renormalized trajectory. This can be understood from the fact that in the limit $N \rightarrow \infty$ the path integral reduces to the classical solution; all fluctuations around the minimum are suppressed with weight zero.

\section{Summary and outlook}

What we should know to determine the classically perfect action for any $N$ is the FPA $\frac{1}{G} S^{*}[\bar{\chi}, \chi, \Phi]$, which is reproduced under RGT. However, this could be obtained analytically only in the small $\Phi$ approximation.

If we let $N \rightarrow \infty$, it suffices to consider a constant $\Phi_{0}$ and $S^{*}$ can be calculated exactly (nonperturbatively). Then the large $N$ limit does a second job for us and makes the line of perfect classical actions coincide with the renormalized trajectory, such that we indeed observe perfect scaling.

For finite $N$, both trajectories will change (there occur higher order interactions etc.) and we have no reason to expect that they still coincide. It would be interesting to determine the size of the cut-off artifacts on the classical trajectory in this case. This could be done either numerically for finite $N$, similar to [1] , or by a $1 / N$ expansion. A basis for the latter is given by our results for the small field approximation applied to the non-zero modes of $\Phi(k)$.

We are confident that our result holds (qualitatively) also for $O(N)$ and $C P(N)$ models. Then for $O(N)$ the success of the work of Hasenfratz

\footnotetext{
${ }^{3}$ Both equations are quite complicated, but they have exactly the same structure, which allows for a drastic simplification.
}

and Niedermayer indicates that the artifacts are still very small at $N=3$.

\section{Conclusions}

At $N \rightarrow \infty$ the procedure of Hasenfratz and Niedermayer leads to a perfect scaling in the Gross-Neveu model, as we showed analytically. In the scaling behavior, cut-off artifacts are completely eliminated at any correlation length. That the FPA really describes continuum physics on a lattice (of finite spacing) can also be seen from the fact that the continuum energy spectrum, and with it continuous Poincaré invariance, are restored.

The small field approximation does not improve the scaling essentially. Here the desired direction out of the critical surface is projected on a subspace, which is too restrictive.

Also the asymptotic scaling is improved for the FPA, but this is not of primary importance.

\section{REFERENCES}

1. P. Hasenfratz, F. Niedermayer, Nucl. Phys. B414 (1994) 785

2. S.-K. Ma, Modern Theory of Critical Phenomena, Benjamin Inc. 1976

3. J. Kogut, K. Wilson, Phys. Rep. C12 (1974) 75

4. A. Hasenfratz, these proceedings

5. W. Bietenholz, E. Focht, U.-J. Wiese, Preprint MIT CTP 2356, HLRZ 35-94 (heplat: 9409018)

6. U.-J. Wiese, Phys. Lett. B315 (1993) 417

7. W. Bietenholz in Proceedings of the XIV Brazilian National Meeting on Particles and Fields (1993) 360 (hep-lat 9402005)

8. W. Bietenholz, U.-J. Wiese, Nucl. Phys. B (Proc. Suppl.) 34 (1994) 516

9. T. Kalkreuter, G. Mack, M. Speh, Int. J. Mod. Phys. C3 (1992) 121

10. L. Bélanger, R. Lacaze, A. Morel, N. Attig, B. Petersson, M. Wolff, Nucl. Phys. B340 (1990) 245 